\title{
Does local factors alter discharge times after surgery?
}

\author{
Mark C. Kendall ${ }^{1}$. Lucas J. Castro-Alves ${ }^{1}$
}

Received: 10 January 2018 / Accepted: 17 February 2018 / Published online: 27 February 2018

(c) Japanese Society of Anesthesiologists 2018

To the Editor:

We read with great interest the article of Nishida et al. [1] in a recent issue of the journal. The authors evaluated patients $<18$ years of age who underwent pediatric ambulatory surgery under general anesthesia and concluded that increased time spent in a hospital was affected by the type of regional block, surgery, and intraoperative fluid volume. The authors should be congratulated for performing a well-designed trial in an important topic in pediatric patients [2]. Moreover, current emphasis on fluid management and regional blocks as a part of enhanced recovery protocols makes the topic more relevant [3].

There are some questions that need to be clarified to determine the validity of the results. First, the authors arbitrarily defined prolonged stay as within $8 \mathrm{~h}$ at the end of anesthesia. It would be important to perform a sensitivity analysis to demonstrate if their results differ when other time cutoffs are utilized (e.g., 6 and 4 h). Second, it is unclear how frequent patients were assessed using the PADSS instrument and if the results can be affected by bias on unstandardized discharge evaluations. Last, most studies examining discharge times demonstrate $<3 \mathrm{~h}$. One could argue that the discharge time in this study is driven by practice characteristics and therefore, poor generalizability to other centers.

Funding No funding was sought.

\section{Compliance with ethical standards}

Conflict of interest The authors declare that they have no conflicts of interest and nothing to disclose.

\section{References}

1. Nishida T, Mihara T, Ka K. Predictors for incidence of increased time spent in hospital after ambulatory surgery in children: a retrospective cohort study. J Anesth. 2017. https://doi.org/10.1007/ s00540-017-2437-9.

2. Cai Y, Lopata L, Roh A, Huang M, Monteleone MA, Wang S, Sun LS. Factors influencing postoperative pain following discharge in pediatric ambulatory surgery patients. J Clin Anesth. 2017;39:100-4.

3. Schol PB, Terink IM, Lancé MD, Scheepers HC. Liberal or restrictive fluid management during elective surgery: a systematic review and meta-analysis. J Clin Anesth. 2016;35:26-39.

This comment refers to the article available at https://doi. org/10.1007/s00540-017-2437-9.

Mark C. Kendall

mark.kendall@lifespan.org

1 Department of Anesthesiology, Rhode Island Hospital,

Warren Alpert Medical School of Brown University, 593

Eddy Street, Providence, RI 02903, USA 